\title{
NEW REGULARITY RESULTS FOR A GENERIC MODEL EQUATION IN EXTERIOR 3D DOMAINS
}

\author{
STANISLAV KRAČMAR \\ Czech Technical University, Faculty of Mech. Engineering, Dept. of Technical Mathematics \\ Karlovo nám. 13, 12135 Praha, Czech Republic \\ E-mail: kracmar@fsik.cvut.cz \\ PATRICK PENEL \\ Université de Toulon et du Var, Department of Mathematics \\ B.P.132, 83957 Toulon - La Garde, France \\ E-mail: penel@univ-tln.fr
}

\begin{abstract}
We consider a generic scalar model for the Oseen equations in an exterior threedimensional domain. We assume the case of a non-constant coefficient function. Using a variational approach we prove new regularity properties of a weak solution whose existence and uniqueness in anisotropically weighted Sobolev spaces were proved in [10]. Because we use some facts and technical tools proved in the above mentioned paper, we give also a brief review of its results and methods.
\end{abstract}

1. Introduction. The classical Oseen equations describe the velocity field and the associated pressure of flow of a viscous fluid past a body by a linearized version of the incompressible Navier-Stokes equations around the velocity at infinity, see [12]. For a compact body $\Omega_{c}$ and the exterior domain $\Omega=\mathbb{R}^{3} \backslash \Omega_{c}$ the system of the Oseen equations can be written in the steady case as

$$
\begin{gathered}
-\nu \Delta v+k \partial_{1} v+\nabla p=F \quad \text { in } \Omega, \\
\nabla \cdot v=G \text { in } \Omega,
\end{gathered}
$$

with the boundary conditions

$$
\begin{gathered}
v=0 \quad \text { on } \partial \Omega_{c}, \\
v \rightarrow 0 \quad \text { as }|x| \rightarrow \infty .
\end{gathered}
$$

2000 Mathematics Subject Classification: 35B40, 35D10, 35J20.

The paper is in final form and no version of it will be published elsewhere. 
$F$ and $G$ are given functions, $\nu$ and $k$ are some positive constants, respectively for the kinematic viscosity and $k=\left|v_{\infty}\right|$ assuming the constant velocity at infinity $v_{\infty}=\left|v_{\infty}\right| e_{1}$ given in the same direction as the first coordinate axis. It is well known that the Oseen fundamental tensor exhibits various decay properties in various directions in $\mathbb{R}^{3}$. A common approach to study the asymptotic properties of the solutions to the Dirichlet problem of the classical steady Oseen flow is to use convolutions with Oseen fundamental tensor and its first and second gradients for the velocity (or with the fundamental solution of Laplace equation for the pressure), see e.g. [4, 3, 8, 9, 7].

Various examples of flows around various obstacles lead us to an interesting variant of equation (1.1):

$$
-\nu \Delta v+k \partial_{1} v+(a \cdot \nabla) v+\nabla p=F \quad \text { in } \Omega .
$$

The additional term here is $(a \cdot \nabla) v, a$ is a concrete non-constant vector function, in the form $\omega \times x$ for a rotating obstacle, see e.g. [5, 11]; in the form of a given "experimental" velocity field for industrial applications [6] when it is impossible to ignore the nonlinear term of the initial Navier-Stokes equations.

Let us introduce a generic scalar model of the equation (1.5) with a given non-constant vector function $a$ :

$$
\begin{gathered}
-\nu \Delta u+k \partial_{1} u+a \cdot \nabla u=f \text { in } \Omega, \\
u=0 \quad \text { on } \partial \Omega_{c}, \\
u \rightarrow 0 \quad \text { as }|x| \rightarrow \infty .
\end{gathered}
$$

As it is well known, in exterior domains, both convective operators $k \partial_{1}$ and $a \cdot \nabla$ cannot be treated as perturbations of lower order of the Laplacian. The study of this model takes also its motivation from the work of Farwig [2]. In the case of a non-constant $a(\cdot)$ we cannot follow it because we have no expression of the fundamental solution. So, in [10] we choose an alternative i.e. a pure variational approach to prove the weak solvability and uniqueness of a solution; this approach works under some appropriate conditions on function $a$.

It is crucial to understand the anisotropic structure of the solutions near the infinity. As a guide, to reflect the decay properties of the fundamental solution in the case $a \equiv 0$, Farwig in $[2,3]$ considers the weight function

$$
\eta_{\beta}^{\alpha}(x)=(1+|x|)^{\alpha}\left(1+|x|-x_{1}\right)^{\beta} .
$$

$\beta$ is the anisotropy exponent. We can always rescale the isotropic and anisotropic parts of $\eta_{\beta}^{\alpha}$ in the form

$$
\begin{gathered}
\eta_{\beta}^{\alpha} \equiv \eta_{\beta}^{\alpha}(\cdot ; \delta, \varepsilon)=(1+\delta r)^{\alpha}(1+\varepsilon s)^{\beta}, \\
\delta, \varepsilon>0, \quad r \equiv r(x)=|x|, \quad s \equiv s(x)=|x|-x_{1} .
\end{gathered}
$$

The natural functional framework for studying the model (1.6)-(1.8) is the corresponding weighted Sobolev spaces. The main results of this paper concern the regularity properties of the weak solution.

The paper is organized as follows. Our notation is standard and requires little explanations in Section 2. Section 3 is devoted to a review of results about existence and unique- 
ness of a weak solution to the problem (1.6)-(1.8) in anisotropically weighted Sobolev spaces, and also to a brief description of the method used in the proof. The main results about regularity properties of these solutions are proved using the variational method in Section 4 . The last Section 5 gives some additional regularity results for a wider range of parameters $\alpha, \beta$, coming back to the case $a \equiv 0$ and using potential theory. Three appendices complete the paper, Appendix A to summarize elementary formulas and some known results used in Sections 4 and 5, Appendix B to recall some known facts (see [2, 3]) we need in Section 5, Appendix $\mathrm{C}$ to discuss some open questions.

2. Basic notations. The domain $\Omega$ is exterior to a body $\Omega_{c}$, i.e. $\Omega=\mathbb{R}^{3} \backslash \Omega_{c}$, we assume $\Omega_{c}$ to be compact, with non-empty interior containing the origin of the Cartesian coordinate system and with a Lipschitz boundary $\partial \Omega_{c}$; we denote by $m>0$ the distance of $\partial \Omega_{c}$ to the origin $\operatorname{dist}\left(0, \partial \Omega_{c}\right)$. Let $M>0$ be a real number such that $B^{M} \subset \Omega$.

We need to denote the special sets: $\Omega^{R}=\Omega \cap B^{R}, \Omega_{R}=\Omega \cap B_{R}$ and $\Omega_{R}^{R_{0}}=\Omega_{R} \cap \Omega^{R_{0}}$, where $B^{R}=\left\{x \in \mathbb{R}^{3},|x|>R\right\}, B_{R}=\left\{x \in \mathbb{R}^{3} ;|x|<R\right\}$ and $B_{R}^{R_{0}}=B^{R_{0}} \cap B_{R}$, for positive numbers $R_{0}<R$.

Let $L^{2}(\Omega ; w)$ be the set of measurable functions $f(\cdot)$ on $\Omega$ such that

$$
\|f\|_{2, \Omega ; w}=\left(\int_{\Omega}|f|^{2} w d x\right)^{1 / 2}<\infty .
$$

We will use the notation $L_{\alpha, \beta}^{2}(\Omega)$ instead of $L^{2}\left(\Omega ; \eta_{\beta}^{\alpha}\right)$ and $\|\cdot\|_{\alpha, \beta}$ or $\|\cdot \mid \Omega\|_{\alpha, \beta}$ instead of $\|\cdot\|_{L^{2}\left(\Omega ; \eta_{\beta}^{\alpha}\right)}$. Let us define the weighted Sobolev space $H^{1}\left(\Omega ; \eta_{\beta_{0}}^{\alpha_{0}}, \eta_{\beta_{1}}^{\alpha_{1}}\right)$ as the set of functions $u \in L_{\alpha_{0}, \beta_{0}}^{2}(\Omega)$ with the weak derivatives $\partial_{i} u \in L_{\alpha_{1}, \beta_{1}}^{2}(\Omega)$. The norm of $u \in H^{1}\left(\Omega ; \eta_{\beta_{0}}^{\alpha_{0}}, \eta_{\beta_{1}}^{\alpha_{1}}\right)$ is given by

$$
\|u\|_{H^{1}\left(\Omega ; \eta_{\beta_{0}}^{\alpha_{0}, \eta_{\beta_{1}}^{\left.\alpha_{1}\right)}}\right.}=\left(\int_{\Omega}|u|^{2} \eta_{\beta_{0}}^{\alpha_{0}} d x+\int_{\Omega}|\nabla u|^{2} \eta_{\beta_{1}}^{\alpha_{1}} d x\right)^{1 / 2} .
$$

As usual, $\stackrel{\circ}{H^{1}}\left(\Omega ; \eta_{\beta_{0}}^{\alpha_{0}}, \eta_{\beta_{1}}^{\alpha_{1}}\right)$ will be the closure of $C_{0}^{\infty}(\Omega)$ in $H^{1}\left(\Omega ; \eta_{\beta_{0}}^{\alpha_{0}}, \eta_{\beta_{1}}^{\alpha_{1}}\right)$.

For simplicity, we shall use the following abbreviations:

$$
\begin{array}{lll}
L_{\alpha, \beta}^{2}(\Omega) & \text { instead of } & L^{2}\left(\Omega ; \eta_{\beta}^{\alpha}\right), \\
\|\cdot\|_{\alpha, \beta} & \text { instead of } & \|\cdot\|_{L^{2}\left(\Omega ; \eta_{\beta}^{\alpha}\right),} \\
\stackrel{\circ}{H}_{\alpha, \beta}^{1}(\Omega) & \text { instead of } & \stackrel{\circ}{H}^{1}\left(\Omega ; \eta_{\beta-1}^{\alpha-1}, \eta_{\beta}^{\alpha}\right), \\
V_{\alpha, \beta}(\Omega) & \text { instead of } & \stackrel{\circ}{H}^{1}\left(\Omega ; \eta_{\beta}^{\alpha-1}, \eta_{\beta}^{\alpha}\right) .
\end{array}
$$

The notations $\stackrel{\circ}{H}^{1}\left(\Omega_{R}\right), H^{k}\left(\Omega_{R}\right)$ are standard without weight. Concerning $V_{\alpha, \beta}(\Omega)$ the weight functions will be taken with appropriate values for rescaling parameters $\delta, \varepsilon$. In formulation of Theorem 3.2: $\delta=\varepsilon=1$.

3. Background. The weak solvability of the problem $(1.6)-(1.8)$ in $V_{\alpha, \beta}(\Omega)$ is established in [10]. The starting point is a usual variational formulation restricted to a bounded domain $\Omega_{R}$. An arbitrary function $\Phi \in \stackrel{\circ}{H}^{1}\left(\Omega_{R}\right)$ can be expressed in the form $\phi \eta_{\beta_{0}}^{0}$ where $\phi \in \stackrel{\circ}{H}^{1}\left(\Omega_{R}\right)$; we consider the following coercive continuous bilinear form on 
$\stackrel{\circ}{H}^{1}\left(\Omega_{R}\right) \times \stackrel{\circ}{H}^{1}\left(\Omega_{R}\right)$ for $\beta_{0}>0$ :

$$
\begin{aligned}
Q_{1}(u, \phi)= & \int_{\Omega_{R}} \nu \cdot \nabla u \cdot \nabla\left(\phi \cdot \eta_{\beta_{0}}^{0}\right) \cdot d x+k \int_{\Omega_{R}} \partial_{1} u \cdot\left(\phi \cdot \eta_{\beta_{0}}^{0}\right) \cdot d x \\
& +\int_{\Omega_{R}}(a \cdot \nabla u)\left(\phi \cdot \eta_{\beta_{0}}^{0}\right) \cdot d x .
\end{aligned}
$$

The coercivity holds under appropriate conditions on $\operatorname{div}\left(\eta_{\beta_{0}}^{0} a\right)$ to control a small negative contribution of the third term in $Q_{1}(u, u)$, but the main tool is the FriedrichsPoincaré type inequality: For $\beta>0$ there are positive constants $R_{0}, c_{0}, c_{1}$ depending on $\alpha, \beta, \delta, \varepsilon$ such that $c_{0}=O\left(\varepsilon^{-2}+\delta^{-2}\right)$ and $c_{1}=O\left(\varepsilon^{-1} \delta^{-1}\right)$ for $\delta$ and $\varepsilon$ tending to zero such that

$$
\|u\|_{\alpha-1, \beta-1}^{2} \leq c_{0}\left\|\nabla u\left|\Omega_{R_{0}}\left\|_{\alpha, \beta}^{2}+c_{1}\right\| \nabla u\right| \Omega^{R_{0}}\right\|_{\alpha, \beta}^{2}
$$

for $u \in \stackrel{\circ}{H}_{\alpha, \beta}^{1}(\Omega)$. In particular, for $\delta=2 \varepsilon$ we get $\|u\|_{\alpha-1, \beta-1} \leq c_{3}\|\nabla u\|_{\alpha, \beta}$, where $c_{3}=\frac{\alpha+\beta}{\beta \beta^{*} \varepsilon}, \beta^{*}=\min (1, \beta)$. For the detailed proof and explicit expressions of constants $c_{0}, c_{1}$ see [10, Lemma 2.3]. We have the following auxiliary result:

Lemma 3.1. Let $0<\beta_{0} \leq 1, f \in L_{1, \beta_{0}}^{2}\left(\Omega_{R}\right)$ and $a \in\left(C^{(1)}\left(\overline{\Omega_{R}}\right)\right)^{3}$. Under appropriate conditions on $\operatorname{div}\left(\eta_{\beta_{0}}^{0} a\right)$, there exists $u_{R} \in \stackrel{\circ}{H}^{1}\left(\Omega_{R}\right)$, the unique weak solution of

$$
Q\left(u_{R}, \Phi\right)=Q_{1}\left(u_{R}, \phi \eta_{\beta_{0}}^{0}\right)=\int_{\Omega_{R}} f \Phi d x
$$

for all $\Phi \in \stackrel{\circ}{H}^{1}\left(\Omega_{R}\right)$.

For the proof see [10, Lem. 3.2, Rem. 3.3]. Function $u_{R}$ satisfies

$$
\begin{gathered}
-\nu \cdot \Delta u_{R}+k \cdot \partial_{1} u_{R}+a \cdot \nabla u_{R}=f \quad \text { in } \Omega_{R}, \\
u_{R}=0 \quad \text { on } \partial \Omega_{R} \equiv \partial \Omega_{c} \cup \partial B_{R} .
\end{gathered}
$$

We can extend $u_{R}$ by zero on $\Omega \backslash \Omega_{R}$, say $\widetilde{u_{R}}$. Uniform estimates as $R \rightarrow \infty$ are necessary to justify the limit case and to find a weak solution to the problem (1.6)-(1.8). To get the uniform boundedness of $\widetilde{u_{R}}$ in $V_{\alpha, \beta}(\Omega)$ as $R \rightarrow \infty$ we need some additional asymptotic conditions on function $a$. It is also necessary to restrict the values of $\alpha / \beta$ by some constant $y_{1} \in(0,1)$. We get the existence and uniqueness theorem. In particular, we proved in [10, Thm. 3.5, Lem. 3.7, Lem. 3.8, Thm. 3.9 i)]:

THEOREM 3.2. Let $0<\beta \leq 1,0 \leq \alpha<y_{1} \cdot \beta, f \in L_{\alpha+1, \beta}^{2}(\Omega)$ and $a \in\left(C^{(1)}(\bar{\Omega})\right)^{3}$. Let $\gamma_{0}=\gamma_{0}(r)$ be a nonnegative non-increasing continuous function defined on interval $[m,+\infty)$ with the limit $\lim _{r \rightarrow+\infty} \gamma_{0}(r)=0$. Further, let $K>0, d>0,0<\nu_{0}<\nu$ and $c \in \mathbb{R}$ such that in $\Omega$ :

$$
a \cdot \frac{\nabla s}{|\nabla s|} \leq K \eta_{-1 / 4+d}^{-3 / 4-d}, \quad \operatorname{div} a \leq \frac{\nu_{0}}{2^{4-\beta}} \frac{1}{r^{2}}, \quad c \leq a \cdot \nabla r \leq \gamma_{0}(r) \eta_{-1}^{0} .
$$

Then there exists a unique weak solution $u \in V_{\alpha, \beta}(\Omega)$ of the problem (1.6)-(1.8) such that

$$
\|u\|_{\alpha-1, \beta}^{2}+\|\nabla u\|_{\alpha, \beta}^{2} \leq C\|f\|_{\alpha+1, \beta}^{2}
$$


REMARK 3.3. It follows from the proof of Theorem 3.2 that without the condition $c \leq$ $a \cdot \nabla r$ it is still possible to prove the existence of a weak solution $u \in V_{\alpha, \beta}(\Omega)$ to (1.6)-(1.8). The additional assumption of the mentioned condition gives the uniqueness of a weak solution in $V_{0, \beta}(\Omega) \supset V_{\alpha, \beta}(\Omega)$.

4. First regularity properties of solutions. In this section we derive some regularity properties of the weak solution given by Theorem 3.2. First we would like to concentrate on the regularity of the solutions near the boundary of the body $\Omega_{c}$.

Our bilinear form $Q(\cdot, \cdot)$ is a particular case of general strongly elliptic form on a closed subspace of $H^{1}\left(\Omega_{R}\right)$. So, the smoothness of every weak solution of $u_{R}$ to the equation (3.2) follows from a general result (see e.g. [14, Thm. 6.C]), we have:

REMARK 4.1. $u_{R_{0}}$ belongs to $H^{2+k}\left(\Omega_{R_{0}}\right)$ if $f \in H^{k}\left(\Omega_{R_{0}}\right)$ assuming the boundary $\partial \Omega_{R_{0}}$ to be a $C^{2+k}$-manifold, and the given function $a \in C^{1+k}\left(\overline{\Omega_{R_{0}}}\right), k \in \mathbb{N}_{0}$. Moreover, there is a constant $C>0$ depending on $R_{0}$ and on $a(\cdot)$, not depending on $f$, such that

$$
\|u\|_{2,2+k} \leq C\|f\|_{2, k},
$$

$\left(\|v\|_{2, k}\right.$ means here the norm in $\left.H^{k}\left(\Omega_{R}\right)\right)$. Let us mention that we will use inequality (4.1) only for fixed bounded domains $\Omega_{R_{0}}$, e.g. $R_{0}:=4 M$.

We assume the following situation: Let $\partial \Omega_{c}$ be of class $C^{2+k}, a \in C^{1+k}\left(\overline{\Omega_{R}}\right)$ and $f \in H^{k}\left(\Omega_{R}\right)$ for some $R \geq M, k \in \mathbb{N}_{0}$. Further let $f \in L_{\alpha+1, \beta}^{2}(\Omega)$ and all the other conditions of Theorem 3.2 be satisfied, such that there exists a weak solution $u_{1} \in V_{\alpha, \beta}(\Omega)$ of the problem (1.6)-(1.8).

Let $t>0$ be given such that $B^{R-2 t} \subset \Omega$, and $\chi_{R}$ be the function from $C_{0}^{\infty}\left(\mathbb{R}^{3}\right)$ such that $\chi_{R} \equiv 1$ in $\Omega_{R-2 t}$, and $\chi_{R} \equiv 0$ in $\Omega^{R-t}$. For an arbitrary $\phi \in C_{0}^{\infty}(\Omega)$ we have:

$$
Q\left(u_{1}, \phi\right)=\int_{\Omega} f \phi d x .
$$

So, we also have:

$$
Q\left(u_{1}, \chi_{R} \phi\right)=\int_{\Omega} f \chi_{R} \phi d x .
$$

Adding to both sides $-2 \nu \int_{\Omega} \nabla u_{1} \cdot \nabla \chi_{R} \phi d x-\nu \int_{\Omega} u_{1} \phi \triangle \chi_{R} d x+k \int_{\Omega} u_{1} \partial_{1} \chi_{R} \phi d x$ $+\int_{\Omega} a \cdot \nabla \chi_{R} \phi u_{1} d x$, we get:

$$
\begin{aligned}
Q\left(\chi_{R} u_{1}, \phi\right)= & \int_{\Omega}\left(f \chi_{R}-2 \nu \nabla u_{1} \cdot \nabla \chi_{R}-\nu u_{1} \triangle \chi_{R}\right) \phi d x \\
& +\int_{\Omega}\left(k u_{1} \partial_{1} \chi_{R}+a \cdot \nabla \chi_{R} u_{1}\right) \phi d x,
\end{aligned}
$$

for an arbitrary $\phi \in C_{0}^{\infty}(\Omega)$, and so also for arbitrary $\phi \in C_{0}^{\infty}\left(\Omega_{R}\right)$. It is clear that $\chi_{R} u_{1} \in \stackrel{\circ}{H}^{1}\left(\Omega_{R}\right)$ is a weak solution of the problem with the right-hand side $F=f \chi_{R}$ $-2 \nu \nabla u_{1} \cdot \nabla \chi_{R}-\nu u_{1} \triangle \chi_{R}+k u_{1} \partial_{1} \chi_{R}+a \cdot \nabla \chi_{R} u_{1}$. From $\chi_{R} u_{1} \in \stackrel{\circ}{H}^{1}\left(\Omega_{R}\right)$ we can see that $F \in L^{2}\left(\Omega_{R}\right)$, and by Remark $4.1 \chi_{R} u_{1} \in H^{2}\left(\Omega_{R}\right)$. Then, using repeatedly Remark 4.1 we get $\chi_{R} u_{1} \in H^{k+2}\left(\Omega_{R}\right)$. So, we proved the following theorem:

THEOREM 4.2. Let all the conditions of Theorem 3.2 be satisfied. Further let $\partial \Omega_{c}$ be of class $C^{2+k}, a \in C^{1+k}\left(\overline{\Omega_{R}}\right)$ and $f \in H^{k}\left(\Omega_{R}\right)$ for some $k \in \mathbb{N}_{0}$ and $R \geq M$. Then the weak 
solution $u$ of the problem (1.6)-(1.8) belongs to $H^{k+2}\left(\Omega_{R-\tau}\right)$, for arbitrary $\tau>0$ such that $B^{M-\tau} \subset \Omega$.

Therefore the local regularity near the boundary of the body depends only on local properties of the right-hand side and on the local behavior of the function $a$.

The next theorem improves the estimate (3.5) from Theorem 3.2 for $\partial_{1} u$ if function $a(\cdot)$ has some additional properties. We now will study properties of the solution separately in the direction of axis $x_{1}$ and the directions of axes $x_{2}, x_{3}$, we introduce the following notation: $a_{*}(x)=\left(a_{2}(x), a_{3}(x)\right), \nabla^{*} v=\left(\partial_{2} v, \partial_{3} v\right)$.

THEOREM 4.3. Let all the conditions of Theorem 3.2 be satisfied (the condition which ensures the uniqueness is not necessary here). Moreover, let $a_{1} \geq-k+\kappa,\left|a_{*}\right| \leq c_{*} \eta_{0}^{-1 / 2}$ in $\Omega$ for some $\kappa>0, c_{*}>0$. Then for every weak solution $u$ of the problem (1.6)-(1.8) we have $\partial_{1} u \in L_{\alpha+1, \beta}^{2}(\Omega)$, and there exists a constant $C>0$ such that

$$
\left\|\partial_{1} u\right\|_{\alpha+1, \beta}^{2} \leq C\|f\|_{\alpha+1, \beta}^{2} .
$$

If additionally $\left|a_{1}\right| \leq c_{1}$ for some $c_{1}>0$, then we have $a \cdot \nabla u \in L_{\alpha+1, \beta}^{2}(\Omega), \triangle u \in$ $L_{\alpha+1, \beta}^{2}(\Omega)$, and there exists a constant always denoted by $C>0$ such that

$$
\|a \cdot \nabla u, \triangle u\|_{\alpha+1, \beta}^{2} \leq C\|f\|_{\alpha+1, \beta}^{2} .
$$

Proof. Let $R>8 M, \phi_{R}=\phi_{R}(r) \in C_{0}^{\infty}([m,+\infty))$ be a cut-off function such that $\phi_{R}(r) \equiv 0$ for $r \leq 2 M$ or $r \geq R,\left(B^{M} \subset \Omega\right), \phi_{R}(r) \equiv 1$ for $r \in[3 M, R / 2]$ and nonincreasing in $[R / 2, R]$. The cut-off function can be chosen such that $-3 / R \leq \phi_{R}^{\prime} \leq 2 / M$.

Let $\Phi_{R} \equiv \Phi_{R}(x) \equiv \phi_{R}(|x|)$. We have $\left|\nabla \Phi_{R}(x)\right| \leq 3 / R$ and $\left|\partial_{1} \Phi_{R}\right| \leq 3 / R$ for $x \in \Omega$, $R / 2 \leq|x| \leq R$.

Because $f \in L_{l o c}^{2}(\Omega)$, we have $u \in H_{l o c}^{2}(\Omega)$, and we may test the equation (1.6) by the function $\left(\partial_{1} u\right) \eta_{\beta}^{\alpha+1} \Phi_{R}^{2}$ :

$$
\begin{aligned}
-\nu \int_{\Omega} \Delta & u\left(\partial_{1} u\right) \eta_{\beta}^{\alpha+1} \Phi_{R}^{2} d x+k \int_{\Omega}\left(\partial_{1} u\right)^{2} \eta_{\beta}^{\alpha+1} \Phi_{R}^{2} d x \\
& +\int_{\Omega} a \cdot \nabla u\left(\partial_{1} u\right) \eta_{\beta}^{\alpha+1} \Phi_{R}^{2} d x=\int_{\Omega} f\left(\partial_{1} u\right) \eta_{\beta}^{\alpha+1} \Phi_{R}^{2} d x .
\end{aligned}
$$

Writing equation (4.4) in the form $I_{\nu}+I_{k}+I_{a}=I_{f}$ we will estimate the first and the third terms on the left-hand side from below and the right-hand side from above:

$$
\begin{aligned}
I_{\nu}= & -\nu \int_{\Omega} \Delta u\left(\partial_{1} u\right) \eta_{\beta}^{\alpha+1} \Phi_{R}^{2} d x=\nu \int_{\Omega} \nabla u \nabla\left(\partial_{1} u \eta_{\beta}^{\alpha+1} \Phi_{R}^{2}\right) d x \\
= & -\frac{\nu}{2} \int_{\Omega}|\nabla u|^{2} \partial_{1}\left(\eta_{\beta}^{\alpha+1} \Phi_{R}^{2}\right) d x+\nu \int_{\Omega} \nabla u \nabla\left(\eta_{\beta}^{\alpha+1} \Phi_{R}^{2}\right) \partial_{1} u d x \\
\geq & -\frac{\nu}{2} \int_{\Omega}|\nabla u|^{2} \partial_{1}\left(\eta_{\beta}^{\alpha+1} \Phi_{R}^{2}\right) d x-c_{4} \int_{\Omega}|\nabla u| \eta_{\beta-1 / 2}^{\alpha+1 / 2}\left|\partial_{1} u\right| \Phi_{R}^{2} d x \\
& -6 \nu \int_{\Omega_{R}^{R / 2}}|\nabla u| \cdot \frac{1}{r}\left|\partial_{1} u\right| \eta_{\beta}^{\alpha+1} \Phi_{R} d x-\frac{2 \nu}{M} \int_{\Omega_{3 M}^{2 M}} \nabla u \partial_{1} u \eta_{\beta}^{\alpha+1} \Phi_{R} d x \\
\geq & -c_{5} \int_{\Omega}|\nabla u|^{2} \eta_{\beta}^{\alpha} d x-\frac{\kappa}{4} \int_{\Omega}\left|\partial_{1} u\right|^{2} \eta_{\beta}^{\alpha+1} \Phi_{R}^{2} d x,
\end{aligned}
$$




$$
\begin{aligned}
I_{a}= & \int_{\Omega} a \cdot \nabla u \partial_{1} u \eta_{\beta}^{\alpha+1} \Phi_{R}^{2} d x \\
= & \int_{\Omega} a_{1}\left(\partial_{1} u\right)^{2} \eta_{\beta}^{\alpha+1} \Phi_{R}^{2} d x+\int_{\Omega}\left(a_{*} \nabla^{*} u\right)\left(\partial_{1} u\right) \eta_{\beta}^{\alpha+1} \Phi_{R}^{2} d x \\
\geq & (-k+\kappa) \int_{\Omega}\left(\partial_{1} u\right)^{2} \eta_{\beta}^{\alpha+1} \Phi_{R}^{2} d x-\frac{\kappa}{4} \int_{\Omega}\left(\partial_{1} u\right)^{2} \eta_{\beta}^{\alpha+1} \Phi_{R}^{2} d x \\
& -\frac{1}{\kappa} \int_{\Omega}\left|\nabla^{*} u\right|^{2} \eta_{\beta}^{\alpha}\left[\left(a_{*}\right)^{2} \eta_{0}^{1}\right] \Phi_{R}^{2} d x \\
\geq & \left(-k+\frac{3 \kappa}{4}\right) \int_{\Omega}\left(\partial_{1} u\right)^{2} \eta_{\beta}^{\alpha+1} \Phi_{R}^{2} d x-\frac{c_{*}^{2}}{\kappa} \int_{\Omega}\left|\nabla^{*} u\right|^{2} \eta_{\beta}^{\alpha} \Phi_{R}^{2} d x, \\
I_{f}= & \int_{\Omega} f\left(\partial_{1} u\right) \eta_{\beta}^{\alpha+1} \Phi_{R}^{2} d x \leq \frac{1}{\kappa} \int_{\Omega} f^{2} \eta_{\beta}^{\alpha+1} \Phi_{R}^{2} d x+\frac{\kappa}{4} \int_{\Omega}\left(\partial_{1} u\right)^{2} \eta_{\beta}^{\alpha+1} \Phi_{R}^{2} d x .
\end{aligned}
$$

The terms containing $\partial_{1} u$ can be absorbed by the second term $I_{k}$ in (4.4), other terms can be estimated by the integral $\int_{\Omega} f^{2} \eta_{\beta}^{\alpha+1} d x$. So, we get:

$$
\frac{\kappa}{4} \int_{\Omega}\left(\partial_{1} u\right)^{2} \eta_{\beta}^{\alpha+1} \Phi_{R}^{2} d x \leq \frac{c_{6}}{\kappa} \int_{\Omega} f^{2} \eta_{\beta}^{\alpha+1} d x .
$$

Using Fatou's lemma, we get for some $c_{7}>0$ :

$$
\int_{\Omega^{3 M}}\left(\partial_{1} u\right)^{2} \eta_{\beta}^{\alpha+1} d x \leq c_{7} \int_{\Omega} f^{2} \eta_{\beta}^{\alpha+1} d x
$$

In a bounded domain e.g. $\Omega_{4 M}$, the estimate of $\int_{\Omega_{4 M}}\left(\partial_{1} u\right)^{2} \eta_{\beta}^{\alpha+1} d x$ is evident with (3.5). Hence, we get (4.2).

We now can observe that $a \cdot \nabla u$ is bounded in $L_{\alpha+1, \beta}^{2}(\Omega)$. Indeed:

$$
\begin{aligned}
\int_{\Omega}(a \cdot \nabla u)^{2} \eta_{\beta}^{\alpha+1} d x & \leq 2 \int_{\Omega} a_{1}^{2}\left|\partial_{1} u\right|^{2} \eta_{\beta}^{\alpha+1} d x+2 \int_{\Omega}\left(a_{*}^{2} \eta_{0}^{1}\right)\left|\nabla^{*} u\right|^{2} \eta_{\beta}^{\alpha} d x \\
& \leq c_{8} \int_{\Omega} f^{2} \eta_{\beta}^{\alpha+1} d x .
\end{aligned}
$$

Directly from the equation (1.6), we obtain the same property for $\triangle u$. Therefore we proved the estimates in (4.3).

As a corollary of the previous theorem we get the estimate of the second order derivatives:

COROLlary 4.4. Let all conditions of Theorem 4.3 be satisfied. Then the following estimate holds for some constant $C>0$ :

$$
\left\|\chi_{0} \nabla^{2} u\right\|_{\alpha+1, \beta}^{2} \leq C\|f\|_{\alpha+1, \beta}^{2},
$$

where $\chi_{0}$ is the characteristic function of any exterior domain $B^{S}$ with $S>0$, such that $B^{S} \subset \Omega$.

Proof. As in the proof of the theorem, we will use the same cut-off function $\Phi_{R} \equiv$ $\Phi_{R}(x) \equiv \phi_{R}(|x|)$. It is enough to estimate $\chi_{0} \nabla^{2} u$ in $\Omega^{3 M}$. Following Farwig we observe that for $\alpha, \beta \in \mathbb{R}$

$$
\left\|\chi_{0} \nabla^{2} u\right\|_{\alpha+1, \beta} \leq C\left(\|\triangle u\|_{\alpha+1, \beta}+\|\nabla u\|_{\alpha, \beta-1}\right) .
$$


Indeed, we have successively:

$$
\begin{aligned}
& \int_{\Omega} \eta_{\beta}^{\alpha+1} \Phi_{R}^{2} \sum_{i, j=1}^{3}\left|\partial_{i} \partial_{j} u\right|^{2} d x \\
& =\int_{\Omega}\left(\eta_{\beta}^{\alpha+1} \Phi_{R}^{2}\right)(\triangle u)^{2} d x+\int_{\Omega} \nabla\left(\eta_{\beta}^{\alpha+1} \Phi_{R}^{2}\right) \cdot \nabla u \triangle u d x \\
& -\sum_{i, j=1}^{3} \int_{\Omega} \partial_{i}\left(\eta_{\beta}^{\alpha+1} \Phi_{R}^{2}\right) \partial_{j} u \cdot \partial_{i} \partial_{j} u d x \\
& \leq \int_{\Omega}(\triangle u)^{2} \eta_{\beta}^{\alpha+1} d x+c_{9} \int_{\Omega} \eta_{\beta-1 / 2}^{\alpha+1 / 2} \Phi_{R}^{2}|\nabla u||\triangle u| d x \\
& +6 \int_{\Omega_{R}^{R / 2}} \frac{1}{r} \cdot|\nabla u||\triangle u| \Phi_{R} \eta_{\beta}^{\alpha+1} d x \\
& +\frac{2}{M} \int_{\Omega_{3 M}^{2 M}}|\nabla u||\triangle u| \Phi_{R} \eta_{\beta}^{\alpha+1} d x \\
& +c_{10} \int_{\Omega} \eta_{\beta-1 / 2}^{\alpha+1 / 2} \Phi_{R}^{2}|\nabla u| \sum_{i, j=1}^{3}\left|\partial_{i} \partial_{j} u\right| d x \\
& +6 \int_{\Omega_{R}^{R / 2}} \frac{1}{r}|\nabla u| \sum_{i, j=1}^{3}\left|\partial_{i} \partial_{j} u\right| \Phi_{R} \eta_{\beta}^{\alpha+1} d x \\
& +\frac{2}{M} \int_{\Omega_{3 M}^{2 M}}|\nabla u| \sum_{i, j=1}^{3}\left|\partial_{i} \partial_{j} u\right| \Phi_{R} \eta_{\beta}^{\alpha+1} d x \\
& \leq \int_{\Omega}(\triangle u)^{2} \eta_{\beta}^{\alpha+1} d x+c_{9} \int_{\Omega} \eta_{\beta-1 / 2}^{\alpha+1 / 2} \Phi_{R}^{2}|\nabla u||\triangle u| d x+c_{11} \int_{\Omega}|\nabla u||\triangle u| \Phi_{R} \eta_{\beta}^{\alpha} d x \\
& +c_{12} \int_{\Omega} \eta_{\beta-1 / 2}^{\alpha+1 / 2} \Phi_{R}^{2}|\nabla u|\left|\nabla^{2} u\right| d x+c_{13} \int_{\Omega}|\nabla u| \cdot\left|\nabla^{2} u\right| \Phi_{R} \eta_{\beta}^{\alpha} d x \\
& \leq c_{14} \int_{\Omega}(\triangle u)^{2} \eta_{\beta}^{\alpha+1} d x+\frac{c_{15}}{t} \int_{\Omega}|\nabla u|^{2} \eta_{\beta-1}^{\alpha} d x+c_{16} t \int_{\Omega}\left|\nabla^{2} u\right|^{2} \eta_{\beta}^{\alpha+1} \Phi_{R}^{2} d x .
\end{aligned}
$$

Taking $t<1 /\left(2 c_{16}\right)$ and subtracting the last term of the right-hand side from left-hand side, we get the inequality (4.6).

THEOREM 4.5. Let the conditions of Theorem 3.2 be satisfied (the condition which ensures the uniqueness is not necessary here). Further let $\partial \Omega_{c}$ be of class $C^{2}, \nabla f \in L_{\alpha+2, \beta+1}^{2}$. Moreover, let $a_{1}<c_{1} \eta_{-1}^{0}$ and always $\left|a_{*}\right| \leq c_{*} \eta_{-1 / 2}^{0}$ in $\Omega$ for some $c_{1}, c_{*}>0$. Then for every weak solution $u$ of the problem (1.6)-(1.8) we have $\nabla u \in L_{\alpha, \beta+1}^{2}(\Omega), \nabla^{2} u \in$ $L_{\alpha+1, \beta+1}^{2}$, and there is constant $C>0$ such that

$$
\left\|\nabla^{2} u\right\|_{\alpha+1, \beta+1}+\|\nabla u\|_{\alpha, \beta+1} \leq C\left(\|f\|_{\alpha+1, \beta}+\|\nabla f\|_{\alpha+2, \beta+1}\right) .
$$

Proof. We will use the same cut-off function as in Theorem 4.3. Because $f \in H^{1}\left(\Omega_{R}\right)$ for an arbitrary $R>M$, and $\partial \Omega_{c} \in C^{2}$, we have $u \in H_{l o c}^{3}(\Omega)$. Then we can multiply the equation (1.6) by $-\nabla\left(\nabla u \eta_{\beta+1}^{\alpha+1} \Phi_{R}^{2}\right)$ and integrate over $\Omega$ : 


$$
\begin{aligned}
-\nu \int_{\Omega} \nabla \Delta & u \nabla u \eta_{\beta+1}^{\alpha+1} \Phi_{R}^{2} d x+k \int_{\Omega} \nabla \partial_{1} u \nabla u \eta_{\beta+1}^{\alpha+1} \Phi_{R}^{2} d x \\
& \quad+\int_{\Omega} \nabla(a \cdot \nabla u) \nabla u \eta_{\beta+1}^{\alpha+1} \Phi_{R}^{2} d x=\int_{\Omega} \nabla f \nabla u \eta_{\beta+1}^{\alpha+1} \Phi_{R}^{2} d x .
\end{aligned}
$$

We estimate the first term on the left-hand side, say $I_{\nu}$ :

$$
\begin{aligned}
I_{\nu}= & -\nu \int_{\Omega} \nabla \Delta u \nabla u \eta_{\beta+1}^{\alpha+1} \Phi_{R}^{2} d x \\
= & \nu \int_{\Omega}(\Delta u)^{2} \eta_{\beta+1}^{\alpha+1} \Phi_{R}^{2} d x+\nu \int_{\Omega} \Delta u \nabla u \nabla\left(\eta_{\beta+1}^{\alpha+1} \Phi_{R}^{2}\right) d x \\
= & \nu \int_{\Omega}(\Delta u)^{2} \eta_{\beta+1}^{\alpha+1} \Phi_{R}^{2} d x \\
& +\nu \int_{\Omega} \Delta u \nabla u \nabla \eta_{\beta+1}^{\alpha+1} \Phi_{R}^{2} d x+\nu \int_{\Omega} \Delta u \nabla u \eta_{\beta+1}^{\alpha+1} \nabla\left(\Phi_{R}^{2}\right) d x
\end{aligned}
$$

By means of the Cauchy-Schwarz inequality, and by the choice of the function $\Phi_{R}($.$) , we$ obtain

$$
\begin{aligned}
I_{\nu} \geq & \frac{\nu}{2} \int_{\Omega}(\Delta u)^{2} \eta_{\beta+1}^{\alpha+1} \Phi_{R}^{2} d x-\nu / 2 \int_{\Omega}(\nabla u)^{2} \frac{\left(\nabla \eta_{\beta+1}^{\alpha+1}\right)^{2}}{\eta_{\beta+1}^{\alpha+1}} \Phi_{R}^{2} d x \\
& -6 \nu \int_{\Omega_{R}^{R / 2}}|\Delta u||\nabla u| \eta_{\beta+1}^{\alpha+1} 2 \Phi_{R} \frac{1}{r} d x-\frac{2}{M} \nu \int_{\Omega_{3 M}^{2 M}}|\Delta u||\nabla u| \eta_{\beta+1}^{\alpha+1} 2 \Phi_{R} d x . c r
\end{aligned}
$$

Let us denote $I_{k}$ the second term on the left-hand side of (4.8), we have:

$$
\begin{aligned}
I_{k}= & -\frac{k}{2} \int_{\Omega}(\nabla u)^{2} \partial_{1}\left(\eta_{\beta+1}^{\alpha+1} \Phi_{R}^{2}\right) d x \\
= & -\frac{k}{2} \int_{\Omega}(\nabla u)^{2} \partial_{1} \eta_{\beta+1}^{\alpha+1} \Phi_{R}^{2} d x-\frac{k}{2} \int_{\Omega}(\nabla u)^{2} \eta_{\beta+1}^{\alpha+1} \partial_{1}\left(\Phi_{R}^{2}\right) d x \\
\geq & -\frac{k}{2} \int_{\Omega}(\nabla u)^{2} \partial_{1} \eta_{\beta+1}^{\alpha+1} \Phi_{R}^{2} d x \\
& -6 \frac{k}{2} \int_{\Omega_{R}^{R / 2}}|\Delta u||\nabla u| \eta_{\beta+1}^{\alpha+1} 2 \Phi_{R} \frac{1}{r} d x-\frac{2}{M} \frac{k}{2} \int_{\Omega_{3 M}^{2 M}}|\Delta u||\nabla u| \eta_{\beta+1}^{\alpha+1} 2 \Phi_{R} d x .
\end{aligned}
$$

As a consequence of these estimates, using the definition (A.6), we get that there exists a constant such that

$$
\begin{aligned}
I_{\nu}+I_{k} \geq & \frac{\nu}{2} \int_{\Omega}(\Delta u)^{2} \eta_{\beta+1}^{\alpha+1} \Phi_{R}^{2} d x+\frac{1}{2} \int_{\Omega}(\nabla u)^{2} F_{\alpha+1, \beta+1}(s, r ; \nu) \eta_{\beta}^{\alpha} \Phi_{R}^{2} d x \\
& -c_{14} \int_{\Omega_{3 M}^{2 M} \cup \Omega_{R}^{R / 2}}|\Delta u||\nabla u| \eta_{\beta+1}^{\alpha} \Phi_{R} d x .
\end{aligned}
$$

We recall in Appendix A how to use function $F_{\alpha, \beta}(s, r ; \nu)$, see Lemma A.2. Hence, if $\kappa>1,0<\varepsilon \leq \frac{1}{2 \kappa} \cdot \frac{k}{\nu} \cdot \frac{\beta-\alpha}{\beta^{2}}$ and $\delta, \nu, k>0$, we obtain:

$$
\begin{aligned}
I_{\nu}+I_{k} & \geq \frac{\nu}{2} \int_{\Omega}(\Delta u)^{2} \eta_{\beta+1}^{\alpha+1} \Phi_{R}^{2} d x-c_{15} \int_{\Omega}|\Delta u||\nabla u| \eta_{\beta+1}^{\alpha} \Phi_{R} d x \\
& +\frac{1}{2} \int_{\Omega}(\nabla u)^{2}\left[\left(1-\frac{1}{\kappa}\right) k \delta \varepsilon(\beta-\alpha) s-(\alpha+1) \delta k\left(1+\frac{\nu}{k}(\alpha+1) \delta\right)\right] \eta_{\beta}^{\alpha} \Phi_{R}^{2} d x .
\end{aligned}
$$


Using again the Cauchy-Schwarz inequality for the second integral with the respective weight functions $\eta_{\beta+1}^{\alpha+1}$ and $\eta_{\beta+1}^{\alpha-1}$, an appropriate choice of the constants and the estimates of Theorem 4.3 lead us to

$$
I_{\nu}+I_{k} \geq \frac{\nu}{4} \int_{\Omega}(\Delta u)^{2} \eta_{\beta+1}^{\alpha+1} \Phi_{R}^{2} d x+c_{16} \int_{\Omega}(\nabla u)^{2} \eta_{\beta+1}^{\alpha} \Phi_{R}^{2} d x-c_{17} \int_{\Omega} f^{2} \eta_{\beta}^{\alpha+1} d x
$$

where $c_{16}=\frac{1}{2}\left(1-\frac{1}{\kappa}\right) k \delta(\beta-\alpha)$.

Coming back to (4.8), we take into account this estimate for $I_{\nu}+I_{k}$ and the following one for the third term in (4.8),

$$
\begin{aligned}
I_{a}= & \int_{\Omega} \nabla(a \cdot \nabla u) \nabla u \eta_{\beta+1}^{\alpha+1} \Phi_{R}^{2} d x \geq-\frac{\nu}{8} \int_{\Omega}(\Delta u)^{2} \eta_{\beta+1}^{\alpha+1} \Phi_{R}^{2} d x \\
& -\frac{c_{16}}{2} \int_{\Omega}(\nabla u)^{2} \eta_{\beta+1}^{\alpha} \Phi_{R}^{2} d x-c_{18} \int_{\Omega} f^{2} \eta_{\beta}^{\alpha+1} d x,
\end{aligned}
$$

therefore we get:

$$
\frac{\nu}{8} \int_{\Omega}(\Delta u)^{2} \eta_{\beta+1}^{\alpha+1} \Phi_{R}^{2} d x+\frac{c_{6}}{4} \int_{\Omega}(\nabla u)^{2} \eta_{\beta+1}^{\alpha} \Phi_{R}^{2} d x \leq c_{19}\left(\int_{\Omega} f^{2} \eta_{\beta}^{\alpha+1} d x+\int_{\Omega}(\nabla f)^{2} \eta_{\beta+1}^{\alpha+2} d x\right) .
$$

The end of the proof looks like the end of the proof of Theorem 4.3: Using the Fatou's lemma the previous estimate gives

$$
\begin{aligned}
\left\|\Delta u\left|\Omega^{3 M}\left\|_{\alpha+1, \beta+1}^{2}+\right\| \nabla u\right| \Omega^{3 M}\right. & \|_{\alpha, \beta+1}^{2} \\
& \leq c_{20}\left(\left\|f\left|\Omega\left\|_{\alpha+1, \beta}^{2}+\right\| \nabla f\right| \Omega\right\|_{\alpha+2, \beta+1}^{2}\right),
\end{aligned}
$$

and in the bounded domain it is clear from (3.5), (4.3) that

$$
\left\|\Delta u\left|\Omega_{4 M}\left\|_{\alpha+1, \beta+1}^{2}+\right\| \nabla u\right| \Omega_{4 M}\right\|_{\alpha, \beta+1}^{2} \leq c_{21}\|f \mid \Omega\|_{\alpha+1, \beta}^{2} .
$$

With (4.11), (4.12), (4.6) and Remark 4.1, we get the inequality (4.7).

To complete the proof, it remains to justify the estimate from below of $I_{a}$ in (4.10):

$$
\begin{aligned}
I_{a}= & -\int_{\Omega}(a \cdot \nabla u) \triangle u \eta_{\beta+1}^{\alpha+1} \Phi_{R}^{2} d x-\int_{\Omega}(a \cdot \nabla u) \nabla u \nabla \eta_{\beta+1}^{\alpha+1} \Phi_{R}^{2} d x \\
& -2 \int_{\Omega}(a \cdot \nabla u) \nabla u \nabla \Phi_{R} \eta_{\beta+1}^{\alpha+1} d x \\
\geq & -\int_{\Omega}\left|a_{1}\right|\left|\partial_{1} u\right||\triangle u| \eta_{\beta+1}^{\alpha+1} \Phi_{R}^{2}-\int_{\Omega}\left|a_{*}\right|\left|\nabla^{*} u\right||\triangle u| \eta_{\beta+1}^{\alpha+1} \Phi_{R}^{2} \\
& -c_{22}\left(\int_{\Omega}\left|a_{1}\right|\left|\partial_{1} u\right||\nabla u| \eta_{\beta+1 / 2}^{\alpha+1 / 2} \Phi_{R}^{2}+\int_{\Omega}\left|a_{*}\right|\left|\nabla^{*} u\right||\nabla u| \eta_{\beta+1 / 2}^{\alpha+1 / 2} \Phi_{R}^{2}\right) \\
& -c_{23}\left(\int_{\Omega}\left|a_{1}\right|\left|\partial_{1} u\right||\nabla u| \Phi_{R} \eta_{\beta+1}^{\alpha}+\int_{\Omega}\left|a_{*}\right|\left|\nabla^{*} u\right||\nabla u| \Phi_{R} \eta_{\beta+1}^{\alpha}\right) .
\end{aligned}
$$

Using the Cauchy-Schwarz inequality for these six integrals, choosing appropriate definitions of the constants, and because of the assumptions on $a_{1}$ and $\left|a_{*}\right|$, we easily obtain the announced estimate.g

COROLlary 4.6. Let the conditions of Theorem 4.5 be satisfied, let $\left|a_{1}\right| \leq c_{1},\left|\nabla a_{1}\right| \leq$ $c \eta_{-1 / 2}^{-1 / 2}$ and $\left|\nabla a_{*}\right| \leq c \eta_{0}^{-1}$. Then for every weak solution $u$ of the problem (1.6)-(1.8) we 
have $\nabla \partial_{1} u \in L_{\alpha+2, \beta+1}^{2}(\Omega), \chi_{0} \nabla^{3} u \in L_{\alpha+2, \beta+1}^{2}(\Omega)$ and there is constant $C>0$ such that

$$
\left\|\nabla \partial_{1} u, \chi_{0} \nabla^{3} u\right\|_{\alpha+2, \beta+1} \leq C\left(\|f\|_{\alpha+1, \beta}+\|\nabla f\|_{\alpha+2, \beta+1}\right) .
$$

Proof. We proceed as in the proofs of Theorem 4.3 and Theorem 4.5: Weakly deriving equation (1.6) by $\nabla$

$$
-\nu \Delta \nabla u+k \partial_{1} \nabla u+\nabla(a \cdot \nabla u)=\nabla f
$$

and testing this equation by $\nabla \partial_{1} u \eta_{\beta+1}^{\alpha+2} \Phi_{R}^{2}$, we get:

$$
\begin{aligned}
-\nu \int_{\Omega} \nabla \Delta u & \nabla \partial_{1} u \eta_{\beta+1}^{\alpha+2} \Phi_{R}^{2} d x+k \int_{\Omega} \nabla \partial_{1} u \nabla \partial_{1} u \eta_{\beta+1}^{\alpha+2} \Phi_{R}^{2} d x \\
& +\int_{\Omega} \nabla(a \cdot \nabla u) \nabla \partial_{1} u \eta_{\beta+1}^{\alpha+2} \Phi_{R}^{2} d x=\int_{\Omega} \nabla f \nabla \partial_{1} u \eta_{\beta+1}^{\alpha+2} \Phi_{R}^{2} d x .
\end{aligned}
$$

The left-hand side of (4.15) can be written as $J_{\nu}+J_{k}+J_{a}$.

We have successively:

$$
\begin{aligned}
J_{\nu}= & -\nu / 2 \int_{\Omega}(\Delta u)^{2} \partial_{1}\left(\eta_{\beta+1}^{\alpha+2} \Phi_{R}^{2}\right) d x+\nu \int_{\Omega} \Delta u \nabla \partial_{1} u \nabla\left(\eta_{\beta+1}^{\alpha+2} \Phi_{R}^{2}\right) d x \\
= & -\nu / 2 \int_{\Omega}(\Delta u)^{2} \partial_{1} \eta_{\beta+1}^{\alpha+2} \Phi_{R}^{2} d x-\nu / 2 \int_{\Omega}(\Delta u)^{2} \eta_{\beta+1}^{\alpha+2} \partial_{1}\left(\Phi_{R}^{2}\right) d x \\
& +\nu \int_{\Omega} \Delta u \nabla \partial_{1} u \nabla \eta_{\beta+1}^{\alpha+2} \Phi_{R}^{2} d x+\nu \int_{\Omega} \Delta u \nabla \partial_{1} u \eta_{\beta+1}^{\alpha+2} \nabla \Phi_{R}^{2} d x \\
\geq & -c_{24} \int_{\Omega}(\Delta u)^{2} \eta_{\beta+1}^{\alpha+1} \Phi_{R}^{2} d x-c_{25} \int_{\Omega}|\Delta u|\left|\nabla \partial_{1} u\right| \eta_{\beta+1 / 2}^{\alpha+3 / 2} \Phi_{R} d x \\
\geq & -c_{24} \int_{\Omega}(\Delta u)^{2} \eta_{\beta+1}^{\alpha+1} \Phi_{R}^{2} d x-\frac{c_{25} t}{2} \int_{\Omega}\left|\nabla \partial_{1} u\right|^{2} \eta_{\beta+1}^{\alpha+2} \Phi_{R}^{2} d x \\
& -\frac{c_{25}}{2 t} \int_{\Omega}(\Delta u)^{2} \eta_{\beta}^{\alpha+1} \Phi_{R} d x \\
\geq & -c_{26}\left(\|f\|_{\alpha+1, \beta}^{2}+\|\nabla f\|_{\alpha+2, \beta+1}^{2}\right)-\frac{\kappa}{2} \int_{\Omega}\left|\nabla \partial_{1} u\right|^{2} \eta_{\beta+1}^{\alpha+2} \Phi_{R}^{2} d x, \\
J_{a}= & \int_{\Omega} \nabla\left(a_{1} \partial_{1} u+a_{*} \nabla^{*} u\right) \nabla \partial_{1} u \eta_{\beta+1}^{\alpha+2} \Phi_{R}^{2} d x \\
\geq & \int_{\Omega} a_{1}\left(\nabla \partial_{1} u\right)^{2} \eta_{\beta+1}^{\alpha+2} \Phi_{R}^{2} d x-\int_{\Omega}\left|a_{*}\right|\left|\nabla^{2} u\right|\left|\nabla \partial_{1} u\right| \eta_{\beta+1}^{\alpha+2} \Phi_{R}^{2} d x \\
& -\int_{\Omega}\left|\nabla a_{1}\right|\left|\partial_{1} u\right|\left|\nabla \partial_{1} u\right| \eta_{\beta+1}^{\alpha+2} \Phi_{R}^{2} d x-\int_{\Omega}\left|\nabla a_{*}\right|\left|\nabla^{*} u\right|\left|\nabla \partial_{1} u\right| \eta_{\beta+1}^{\alpha+2} \Phi_{R}^{2} d x .
\end{aligned}
$$

Using the assumptions on $a_{1},\left|a_{*}\right|,\left|\nabla a_{1}\right|$ and $\left|\nabla a_{*}\right|$, we easily obtain

$$
J_{\nu}+J_{k}+J_{a} \geq \frac{\kappa}{2} \int_{\Omega}\left|\nabla \partial_{1} u\right|^{2} \eta_{\beta+1}^{\alpha+2} \Phi_{R}^{2} d x-c_{27}\left(\|f\|_{\alpha+1, \beta}^{2}+\|\nabla f\|_{\alpha+2, \beta+1}^{2}\right)
$$

and then

$$
\frac{\kappa}{4} \int_{\Omega}\left|\nabla \partial_{1} u\right|^{2} \eta_{\beta+1}^{\alpha+2} \Phi_{R}^{2} d x \leq c_{28}\left(\|f\|_{\alpha+1, \beta}^{2}+\|\nabla f\|_{\alpha+2, \beta+1}^{2}\right)
$$


We have also

$$
\nabla(a \cdot \nabla u)=\partial_{j} a_{1} \partial_{1} u+\partial_{j} a_{k} \partial_{k} u+a_{1} \partial_{j} \partial_{1} u+a_{k} \partial_{j} \partial_{k} u,
$$

where $i, j \in\{1,2,3\}, k \in\{2,3\}$. Then $\nabla(a \cdot \nabla u) \in L_{\alpha+2, \beta+1}^{2}(\Omega)$. So, from the equation (4.14), we get also $\Delta \nabla u \in L_{\alpha+2, \beta+1}^{2}(\Omega)$.

Use of relation (4.6) and Remark 4.1 completes the proof of (4.13).

5. Other regularity properties. To get other regularity properties of solutions we must refer to the results about strong solutions of the equation (1.6) with $a \equiv 0$ derived by the potential theory (see Farwig in $[2,3]$ and Appendix B with $b \equiv 0$ on $\partial \Omega_{c}$ ). Using this approach we can extend the regularity results also on the case $\alpha \geq y_{1} \beta$, and this gives us the possibility to compare the regularity results derived by variational approach and corresponding conditions on function $a$ with results and conditions derived now.

Let the conditions of Theorem 3.2 and Theorem 4.3 be satisfied for the vector function $a($.$) and let us denote by u_{a}$ the unique weak solution in $V_{\alpha, \beta}$ of our problem. Then $a \cdot \nabla u_{a} \in L_{\alpha+1, \beta}^{2}(\Omega):$ This fact is ensured by the conditions on $a($.$) in \Omega,\left|a_{1}\right| \leq c_{1},\left|a_{*}\right| \leq$ $c_{*} \eta_{0}^{-1 / 2}$, and for all $f \in L_{\alpha+1, \beta}^{2}(\Omega)$ with $\alpha, \beta$ such that $0<\beta \leq 1,0 \leq \alpha<y_{1} \cdot \beta$.

With $F=f-a \cdot \nabla u_{a} \in L_{\alpha+1, \beta}^{2}(\Omega)$, equation (1.6) looks like $-\nu \Delta u+k \partial_{1} u=F$ : Its solvability and uniqueness follow from Appendix B, Theorem B.1, let $u_{0, a}$ be the strong solution. This solution $u_{0, a}$ is at the same time the weak solution of our problem in $V_{\alpha, \beta}(\Omega)$. Using the uniqueness argument, we get $u_{a}=u_{0, a}$. So, we have a stronger result for the second order derivatives of $u=u_{a}=u_{0, a}$ than in Theorem 4.3, we get:

$$
\left\|\nabla^{2} u\right\|_{\alpha+1, \beta}^{2} \leq C\|f\|_{\alpha+1, \beta}^{2} .
$$

Assuming once more our problem with $a \not \equiv 0$ in $\Omega$. Let $f \in L_{\alpha+1, \beta}^{2}$ with $\alpha, \beta$ such that $0 \leq \alpha<\beta<1$, i.e. as in Appendix B, Theorem B.1, but with non-negative $\alpha$; when $\alpha \geq y_{1} \beta$, for all $t \in\left(0, y_{1} \beta\right)$, we have $f \in L_{\alpha+1, \beta}^{2} \subset L_{y_{1} \beta+1-t, \beta}^{2}$. Further let the conditions of Theorem 3.2 and Theorem 4.3 be satisfied for $a($.$) , and let us denote$ always by $u_{a}$ the unique weak solution in $V_{y_{1} \beta-t, \beta}(\Omega)$. From Theorem 4.3 it follows that $\partial_{1} u_{a} \in L_{y_{1} \beta+1-t, \beta}^{2}(\Omega)$ and $\nabla u_{a} \in L_{y_{1} \beta-t, \beta}^{2}(\Omega)$. Assuming stronger additional conditions on $a($.$) in \Omega$

$$
\left|a_{1}\right| \leq c_{1} \eta_{0}^{-q}, \quad\left|a_{*}\right| \leq c_{* *} \eta_{0}^{-1 / 2-q},
$$

where $q=\alpha-y_{1} \beta+t$, we get $a \cdot \nabla u_{a} \in L_{\alpha+1, \beta}^{2}(\Omega)$.

Let us consider again $F=f-a \cdot \nabla u_{a}$, and $u_{0, a}$ the strong solution of the problem rewritten in the previous form, see Appendix B, Theorem B.1. This solution $u_{0, a}$ is at the same time the weak solution of our problem in $V_{y_{1} \beta-t, \beta}(\Omega)$. Using the uniqueness argument, we get $u_{a}=u_{0, a}$. So, also in this case we have for the second order derivatives of $u=u_{a}=u_{0, a}$ :

$$
\left\|\nabla^{2} u\right\|_{\alpha+1, \beta}^{2} \leq C\|f\|_{\alpha+1, \beta}^{2}
$$

This implies the following result:

THEOREM 5.1. Let $\Omega \subset \mathbb{R}^{3}$ be an exterior domain with boundary of class $C^{2}$. Further let $0 \leq \alpha<\beta<1, f \in L_{\alpha+1, \beta}^{2}(\Omega)$, and $a \in\left(C^{(1)}(\bar{\Omega})\right)^{3}$ has all properties formulated in 
Theorem 3.2. Let additionally the following assumptions be satisfied in $\Omega$ :

$$
\left|a_{1}\right| \leq c_{1} \eta_{0}^{-q}, \quad\left|a_{*}\right| \leq c_{* *} \eta_{0}^{-1 / 2-q},
$$

where $q=\left\{\begin{array}{cc}\alpha-y_{1} \beta+t, & \text { if } \alpha \geq y_{1} \beta \\ 0, & \text { if } \alpha<y_{1} \beta\end{array}\right.$ for some $t \in\left(0, y_{1} \beta\right)$. Then there exists a strong solution $u \in V_{\alpha, \beta}(\Omega) \cap H_{l o c}^{2}(\bar{\Omega})$ of the problem

$$
\begin{gathered}
-\nu \Delta u+k \partial_{1} u+a \cdot \nabla u=f \text { in } \Omega, \\
u=0 \quad \text { on } \partial \Omega_{c}, \\
u \rightarrow 0 \quad \text { as }|x| \rightarrow \infty
\end{gathered}
$$

such that

$$
\|u\|_{\alpha-1, \beta}^{2}+\|\nabla u\|_{\alpha, \beta}^{2}+\left\|\partial_{1} u, a \cdot \nabla u, \nabla^{2} u\right\|_{\alpha+1, \beta}^{2} \leq C\|f\|_{\alpha+1, \beta}^{2} .
$$

Analogously, using Theorem B.2 and Theorem B.3 the following results can be derived: THEOREM 5.2. Let $\Omega \subset \mathbb{R}^{3}$ be an exterior domain with boundary of class $C^{3}$. Further let $0 \leq \alpha<\beta<1, f \in L_{\alpha+1, \beta}^{2}(\Omega), \nabla f \in L_{\alpha+2, \beta+1}^{2}(\Omega)$, and $a \in\left(C^{(1)}(\bar{\Omega})\right)^{3}$ has all properties formulated in Theorem 3.2. Let additionally the following assumptions for $a($.$) be satisfied$ in $\Omega$ :

$$
\begin{gathered}
\left|a_{1}\right| \leq c_{1} \min \left\{\eta_{0}^{-q}, \eta_{-1 / 2}^{-1 / 2}\right\}, \quad\left|a_{*}(x)\right| \leq c_{*} \min \left\{\eta_{0}^{-1 / 2-q}, \eta_{-1 / 2}^{-1 / 2}\right\}, \\
\left|\nabla a_{1}\right| \leq c \eta_{-1 / 2}^{-1 / 2}, \quad\left|\nabla a_{*}\right| \leq c \eta_{-1 / 2}^{-1}
\end{gathered}
$$

where $q=\left\{\begin{array}{cc}\alpha-y_{1} \beta+t, & \text { if } \alpha \geq y_{1} \beta \\ 0, & \text { if } \alpha<y_{1} \beta\end{array}\right.$ for some $t \in\left(0, y_{1} \beta\right)$. Then there exists a strong solution $u$ of the problem (5.1)-(5.3) such that $u \in L_{\alpha-1, \beta}^{2}(\Omega), \nabla u \in L_{\alpha, \beta+1}^{2}(\Omega), \nabla^{2} u \in$ $L_{\alpha+1, \beta+1}^{2}(\Omega), \partial_{1} \nabla u \in L_{\alpha+2, \beta+1}^{2}(\Omega), \nabla^{3} u \in L_{\alpha+2, \beta+1}^{2}(\Omega)$. And there is a constant $C>0$ such that

$$
\begin{gathered}
\|u\|_{\alpha-1, \beta}^{2}+\|\nabla u\|_{\alpha, \beta+1}^{2}+\left\|\nabla^{2} u\right\|_{\alpha+1, \beta+1}^{2}+\left\|\partial_{1} \nabla u, \nabla^{3} u\right\|_{\alpha+2, \beta+1}^{2} \\
\leq C\left(\|f\|_{\alpha+1, \beta}^{2}+\|\nabla f\|_{\alpha+2, \beta+1}^{2}\right) .
\end{gathered}
$$

THEOREM 5.3. Let $\Omega \subset \mathbb{R}^{3}$ be an exterior domain with boundary of class $C^{3}$. Further let $0 \leq \alpha<\beta<1, f \in L_{\alpha+1, \beta}^{2}(\Omega), \partial_{1} f \in L_{\alpha+3, \beta}^{2}(\Omega)$, and $a \in\left(C^{(1)}(\bar{\Omega})\right)^{3}$ has all properties formulated in Theorem 3.2. Let additionally the following assumptions for a(.) be satisfied in $\Omega$ :

$$
\begin{gathered}
\left|a_{1}\right| \leq c_{1} \eta_{0}^{-1}, \quad\left|a_{*}(x)\right| \leq c_{*} \min \left\{\eta_{0}^{-1 / 2-q}, \eta_{0}^{-1}\right\}, \\
\left|\partial_{1} a_{1}\right| \leq c \eta_{0}^{-1}, \quad\left|\partial_{1} a_{*}\right| \leq c \eta_{0}^{-3 / 2}
\end{gathered}
$$

where $q=\left\{\begin{array}{cc}\alpha-y_{1} \beta+t, & \text { if } \alpha \geq y_{1} \beta \\ 0, & \text { if } \alpha<y_{1} \beta\end{array}\right.$ for some $t \in\left(0, y_{1} \beta\right)$. Then there exists a strong solution $u$ of the problem (5.1)-(5.3) such that $u \in L_{\alpha-1, \beta}^{2}(\Omega), \nabla \partial_{1} u \in L_{\alpha+2, \beta}^{2}(\Omega)$, $\partial_{1}^{2} u \in L_{\alpha+3, \beta}^{2}(\Omega), \nabla^{2} \partial_{1} u \in L_{\alpha+3, \beta}^{2}(\Omega)$. And there is a constant $C>0$ such that

$$
\left\|\nabla \partial_{1} u\right\|_{\alpha+2, \beta}^{2}+\left\|\partial_{1}^{2} u, \nabla^{2} \partial_{1} u\right\|_{\alpha+3, \beta}^{2} \leq C\left(\|f\|_{\alpha+1, \beta}^{2}+\left\|\partial_{1} f\right\|_{\alpha+3, \beta}^{2}\right) .
$$

THEOREM 5.4. Let $\Omega \subset \mathbb{R}^{3}$ be an exterior domain with boundary of class $C^{3}$. Further let $0 \leq \alpha<\beta<1, f \in L_{\alpha+1, \beta}^{2}(\Omega), \nabla f \in L_{\alpha+2, \beta+1}^{2}(\Omega), \partial_{1} f \in L_{\alpha+3, \beta}^{2}(\Omega)$, and $a \in\left(C^{(1)}(\bar{\Omega})\right)^{3}$ 
has all properties formulated in Theorem 3.2. Let additionally the following assumptions for $a($.$) be satisfied in \Omega$ :

$$
\begin{gathered}
\left|a_{1}\right| \leq c_{1} \min \left\{\eta_{0}^{-q}, \eta_{-1 / 2}^{-1 / 2}\right\}, \quad\left|a_{*}(x)\right| \leq c_{*} \min \left\{\eta_{0}^{-1 / 2-q}, \eta_{-1 / 2}^{-1 / 2}\right\} \\
\left|\partial_{1} a_{1}\right| \leq c \eta_{0}^{-1}, \quad\left|\nabla^{*} a_{1}\right| \leq c \eta_{-1 / 2}^{-1 / 2} \\
\left|\partial_{1} a_{*}\right| \leq c \eta_{0}^{-3 / 2} \quad\left|\nabla^{*} a_{*}\right| \leq c \eta_{-1 / 2}^{-1}
\end{gathered}
$$

where $q=\left\{\begin{array}{cc}\alpha-y_{1} \beta+t, & \text { if } \alpha \geq y_{1} \beta \\ 0, & \text { if } \alpha<y_{1} \beta\end{array}\right.$ for some $t \in\left(0, y_{1} \beta\right)$. Then there exists a strong solution $u$ of the problem (5.1)-(5.3) such that $u \in L_{\alpha-1, \beta}^{2}(\Omega), \nabla \partial_{1} u \in L_{\alpha+2, \beta+1}^{2}(\Omega)$, $\partial_{1}^{2} u \in L_{\alpha+3, \beta}^{2}(\Omega), \nabla^{2} \partial_{1} u \in L_{\alpha+3, \beta}^{2}(\Omega)$. And there is a constant $C>0$ such that

$$
\begin{aligned}
\|u\|_{\alpha-1, \beta}^{2}+ & \|\nabla u\|_{\alpha, \beta+1}^{2}+\left\|\nabla^{2} u\right\|_{\alpha+1, \beta+1}^{2}+\left\|\partial_{1} \nabla u, \nabla^{3} u\right\|_{\alpha+2, \beta+1}^{2} \\
& +\left\|\partial_{1}^{2} u, \nabla^{2} \partial_{1} u\right\|_{\alpha+3, \beta}^{2} \leq C\left(\|f\|_{\alpha+1, \beta}^{2}+\|\nabla f\|_{\alpha+2, \beta+1}^{2}+\left\|\partial_{1} f\right\|_{\alpha+3, \beta}^{2}\right) .
\end{aligned}
$$

Appendix A. Obviously we have the explicit expressions:

$$
\partial_{i} r \equiv \frac{\partial r}{\partial x_{i}}=\frac{x_{i}}{r}, \quad \partial_{1} s=\frac{-s}{r},
$$

$$
\nabla r \cdot \nabla r \equiv 1, \quad \nabla s \cdot \nabla r=\frac{s}{r}, \quad \nabla s \cdot \nabla s=\frac{2 s}{r}
$$

$$
\Delta r=\frac{2}{r}, \quad \Delta s=\frac{2}{r},
$$

$$
\begin{gathered}
\partial_{1} \eta_{\beta}^{\alpha}=\left\{\alpha \cdot \delta(1+\varepsilon \cdot s) \frac{x_{1}}{r}-\beta \cdot \varepsilon(1+\delta \cdot r) \frac{s}{r}\right\} \eta_{\beta-1}^{\alpha-1}, \\
\partial_{i} \eta_{\beta}^{\alpha}=\{\alpha \cdot \delta(1+\varepsilon \cdot s)+\beta \cdot \varepsilon(1+\delta \cdot r)\} \frac{x_{i}}{r} \eta_{\beta-1}^{\alpha-1}, \quad i=2,3, \\
\nabla \eta_{\beta}^{\alpha}=\{\alpha \cdot \delta(1+\varepsilon \cdot s) \cdot \nabla r+\beta \cdot \varepsilon(1+\delta \cdot r) \cdot \nabla s\} \eta_{\beta-1}^{\alpha-1}, \\
\left|\nabla \eta_{\beta}^{\alpha}\right|^{2}=\left\{\alpha^{2} \delta^{2}\left(\frac{1+\varepsilon \cdot s}{1+\delta \cdot r}\right)+2 \alpha \beta \delta \varepsilon \frac{s}{r}+2 \beta^{2} \varepsilon^{2}\left(\frac{1+\delta \cdot r}{1+\varepsilon \cdot s}\right) \frac{s}{r}\right\} \cdot\left(\eta_{\beta-1 / 2}^{\alpha-1 / 2}\right)^{2}, \\
\Delta \eta_{\beta}^{\alpha}=\left\{\alpha(\alpha-1) \delta^{2}\left(\frac{1+\varepsilon \cdot s}{1+\delta \cdot r}\right)+2 \alpha \beta \delta \varepsilon \frac{s}{r}\right. \\
\left.+2 \beta(\beta-1) \varepsilon^{2}\left(\frac{1+\delta \cdot r}{1+\varepsilon \cdot s}\right) \frac{s}{r}+2 \alpha \delta(1+\varepsilon \cdot s) \frac{1}{r}+2 \beta \varepsilon(1+\delta \cdot r) \frac{1}{r}\right\} \cdot \eta_{\beta-1}^{\alpha-1} .
\end{gathered}
$$

Because $\left(\eta_{\beta}^{\alpha}\right)^{-1}$ is locally integrable, then, by Hölder's inequality, it follows that $L_{\alpha, \beta}^{2}(\Omega) \subset L_{l o c}^{1}(\Omega)$. It thus makes sense to talk about weak derivatives of functions in $L_{\alpha, \beta}^{2}(\Omega)$.

Let us mention that $\eta_{\beta}^{\alpha}$ belongs to the Muckenhoupt class $A_{2}$ of weights in $\mathbb{R}^{3}$ if $-1<\beta<1$ and $-3<\alpha+\beta<3$. The following proposition is proved exactly in the same way as in the non-weighted case (see R. A. Adams [1, pp. 45-46]), when $\eta_{\beta}^{\alpha} \in A_{2}$.

Lemma A.1. Let $\Omega \subset \mathbb{R}^{3}$ be open set and $\eta_{\beta_{0}}^{\alpha_{0}}, \eta_{\beta_{1}}^{\alpha_{1}} \in A_{2}$. Then $H^{1}\left(\Omega ; \eta_{\beta_{0}}^{\alpha_{0}}, \eta_{\beta_{1}}^{\alpha_{1}}\right)$ and $\stackrel{\circ}{H}^{1}\left(\Omega ; \eta_{\beta_{0}}^{\alpha_{0}}, \eta_{\beta_{1}}^{\alpha_{1}}\right)$ are Banach spaces, more precisely Hilbert spaces equipped with the scalar product

$$
(u, v)_{H^{1}\left(\Omega ; \eta_{\beta_{0}}^{\alpha_{0}}, \eta_{\beta_{1}}^{\alpha_{1}}\right)}=\int_{\Omega} u \cdot v \cdot \eta_{\beta_{0}}^{\alpha_{0}} \cdot d x+\int_{\Omega} \nabla u \cdot \nabla v \cdot \eta_{\beta_{2}}^{\alpha_{1}} \cdot d x
$$


Let us define a function $F_{\alpha, \beta}(s, r ; \nu)$ by the relation:

$$
F_{\alpha, \beta}(s, r ; \nu) \cdot \eta_{\beta-1}^{\alpha-1} \equiv-\nu \frac{\left|\nabla \eta_{\beta}^{\alpha}\right|^{2}}{\eta_{\beta}^{\alpha}}-k \partial_{1} \eta_{\beta}^{\alpha}
$$

The following lemma gives the estimate of $F(s, r ; \nu)$ from below.

LemmA A.2. Let $0 \leq \alpha<\beta, \kappa>1,0<\varepsilon \leq \frac{1}{2 \kappa} \cdot \frac{k}{\nu} \cdot \frac{\beta-\alpha}{\beta^{2}}$ and $\delta, \nu, k>0$. Then

$$
F_{\alpha, \beta}(s, r ; \nu)-\left(1-\frac{1}{\kappa}\right) \cdot k \cdot \delta \cdot \varepsilon \cdot(\beta-\alpha) \cdot s \geq-\alpha \delta k\left(1+\frac{\nu}{k} \alpha \delta\right)
$$

for all $r>0$ and $s \in[0,2 r]$.

For the proof see [10, Lem. 3.1].

Appendix B. The formulations of theorems used in Section 5. The respective potential theory using the fundamental solution was done in [3]. The results was proved for the Oseen system. Due to the identical properties of fundamental solutions one can get analogous theorems also for the model equation. In this section we shall use the following notation:

$$
H_{l o c}^{k}(\bar{\Omega})=\left\{v \in H_{l o c}^{k}(\Omega), v \in H^{k}\left(\overline{\Omega_{R}}\right) \text { for all } R>M\right\}
$$

We used the following theorems in Section 5:

TheOREM B.1 (Farwig). Let $\Omega \subset \mathbb{R}^{3}$ be an exterior domain with boundary of class $C^{2}$. Further let $\alpha, \beta \in \mathbb{R}$ be such that $0 \leq|\alpha|<\beta<1$. Then for all $F \in L_{\alpha+1, \beta}^{2}(\Omega)$ and all boundary values $b \in H^{3 / 2}(\partial \Omega)$ there is a unique strong solution $u \in L_{\alpha-1, \beta}^{2}(\Omega) \cap H_{\text {loc }}^{2}(\bar{\Omega})$ of the problem:

$$
\begin{gathered}
-\nu \Delta u+k \partial_{1} u=F \quad \text { in } \Omega \\
u=b \quad \text { on } \partial \Omega \\
u \rightarrow 0 \quad \text { as }|x| \rightarrow \infty
\end{gathered}
$$

There is a constant $c>0$ independent of $F$ and $b$ such that

$$
\|u\|_{\alpha-1, \beta}+\|\nabla u\|_{\alpha, \beta}+\left\|\partial_{1} u, \nabla^{2} u\right\|_{\alpha+1, \beta} \leq c\left(\|F\|_{\alpha+1, \beta}+\|b\|_{H^{3 / 2}(\partial \Omega)}\right) .
$$

For the proof see [3, Th. 5.6, Rem. 2.9].

Theorem B.2 (Farwig). Let $\Omega \subset \mathbb{R}^{3}$ be an exterior domain with boundary of class $C^{3}$. Further let $\alpha, \beta \in \mathbb{R}$ be such that $0 \leq|\alpha|<\beta<1$. Then for all $F \in L_{\alpha+1, \beta}^{2}(\Omega), \nabla F \in$ $L_{\alpha+2, \beta+1}^{2}(\Omega)$ and all boundary values $b \in H^{5 / 2}(\partial \Omega)$ there is a unique strong solution $u$ of the problem (B.1)-(B.3) such that $u \in L_{\alpha-1, \beta}^{2}(\Omega), \nabla u \in L_{\alpha, \beta+1}^{2}(\Omega), \nabla^{2} u \in L_{\alpha+1, \beta+1}^{2}(\Omega)$, $\partial_{1} \nabla u \in L_{\alpha+2, \beta+1}^{2}(\Omega), \nabla^{3} u \in L_{\alpha+2, \beta+1}^{2}(\Omega)$. There is a constant $c>0$ independent of $F$ and $b$ such that

$$
\begin{array}{r}
\|u\|_{\alpha-1, \beta}+\|\nabla u\|_{\alpha, \beta+1}+\left\|\nabla^{2} u\right\|_{\alpha+1, \beta+1}+\left\|\partial_{1} \nabla u, \nabla^{3} u\right\|_{\alpha+2, \beta+1} \\
\leq c\left(\|F\|_{\alpha+1, \beta}+\|\nabla F\|_{\alpha+2, \beta+1}+\|b\|_{H^{5 / 2}(\partial \Omega)}\right) .
\end{array}
$$

For the proof see $[3$, Th. 5.7 (ii)]. 
TheOREM B.3 (Farwig). Let $\Omega \subset \mathbb{R}^{3}$ be an exterior domain with boundary of class $C^{3}$. Further let $\alpha, \beta \in \mathbb{R}$ be such that $0 \leq|\alpha|<\beta<1$. Then for all $F \in L_{\alpha+1, \beta}^{2}(\Omega)$, $\partial_{1} F \in L_{\alpha+3, \beta}^{2}(\Omega)$ and $b=0$. Then the solution $u$ of the problem (B.1)-(B.3) satisfies

$\left\|\nabla \partial_{1} u\right\|_{\alpha+2, \beta}+\left\|\partial_{1}^{2} u, \nabla^{2} \partial_{1} u\right\|_{\alpha+3, \beta} \leq c\left(\|F\|_{\alpha+1, \beta}+\left\|\partial_{1} F\right\|_{\alpha+3, \beta}\right)$.

For the proof see [2, Th. 3.9 (i)].

Appendix C. In this section we would like to mention formal connections between our case and the the modified Oseen equations with some open questions. Let us assume for simplicity the case $\Omega=\mathbb{R}^{3}$. The modified Oseen equations $(1.5),(1.2)$ can be formally written in the form

$$
\left[\begin{array}{cc}
-\nu \triangle+k \partial_{1}+a \cdot \nabla, & \nabla \\
L_{a}, & -\triangle
\end{array}\right]\left[\begin{array}{l}
u \\
p
\end{array}\right]=\left[\begin{array}{c}
F \\
G_{a}
\end{array}\right],
$$

where $L_{a}=-\sum_{i, j=1}^{3} \partial_{i} a_{j} \partial_{j} u_{i}$ and $G_{a}=-\nabla \cdot F+\left(-\nu \triangle+k \partial_{1} k+a \cdot \nabla\right) G$. As usual we take the divergence of equation (1.5) to determine pressure $p$.

In the case $a \equiv 0$ we have also $L_{a} u=0$ therefore (C.1) is reduced to

$$
\left[\begin{array}{cc}
-\nu \triangle+k \partial_{1}+a \cdot \nabla, & \nabla \\
0, & -\triangle
\end{array}\right]\left[\begin{array}{l}
u \\
p
\end{array}\right]=\left[\begin{array}{c}
F \\
G_{0}
\end{array}\right] .
$$

As we can see, the first three equations of the system are scalar and depend only on $p$ :

$$
-\nu \triangle u_{i}+k \partial_{1} u_{i}+a \cdot \nabla u_{i}=F_{i}-\partial_{i} p, \quad i=1,2,3 .
$$

Given the $F$ and $G$ we solve the equation $-\triangle p=G_{0}$ and then (C.3), each equation being one model scalar model equation with $a \equiv 0$. We can see that the case $a \not \equiv 0$ is essentially different, and the question how to modify the mentioned procedure in the case $a \not \equiv 0$ is still open.

Acknowledgement. The authors gratefully acknowledge and appreciate the hospitality of the Institute of Mathematics of the Polish Academy of Sciences and the University in Toulon. The first author is supported by grant 201/05/0005 from the Grant Agency of the Czech Republic, partly supported by grant IAA2120201/02 from the Academy of Sciences of the Czech Republic and by the project MSM 6840770010 of the Ministry of Education of the Czech Republic.

\section{References}

[1] R. A. Adams, Sobolev Spaces, Academic Press, New York, 1975.

[2] R. Farwig, A variational approach in weighted Sobolev spaces to the operator $-\triangle+\partial / \partial_{1}$ in exterior domains of $\mathbb{R}^{3}$, Mathematische Zeitschrift 210 (1992), 449-464.

[3] R. Farwig, The stationary exterior 3-D problem of Oseen and Navier-Stokes equations in anisotropically weighted Sobolev spaces, Mathematische Zeitschrift 211 (1992), 409-447.

[4] R. Finn, Estimates at infinity for stationary solution of Navier-Stokes equations, Bull. Math. de la Soc. Sci. Math. de la R.P.R. 3 (51) (1959), 387-418.

[5] T. Hishida, The Stokes operator with rotation effect in exterior domains, Analysis 19 (1999), 51-67. 
[6] J.-L. Impagliazzo, Résolution des équations de Navier-Stokes compressibles à l'aide de la méthode de décomposition, Thèse de doctorat de l'Université de Toulon et du Var, 1997.

[7] T. Kobayashi and Y. Shibata, On the Oseen equation in the three-dimensional exterior domains, Math. Ann. 310 (1998), 1-45.

[8] S. Kračmar, A. Novotný and M. Pokorný, Estimates of three dimensional Oseen kernels in weighted $L^{p}$ spaces, in: Applied Nonlinear Analysis, Plenum Publishers/Kluwer Academic, London-New York, 1999, 281-316.

[9] S. Kračmar, A. Novotný and M. Pokorný, Estimates of Oseen kernels in weighted $L^{p}$ spaces, J. Math. Soc. Japan 53 (2001), 59-111.

[10] S. Kračmar and P. Penel, Variational properties of a generic model equation in exterior $3 D$ domains, Funkcial. Ekvac. 47 (2004), 499-523.

[11] Š. Nečasová, Asymptotic properties of the steady fall of a body in a viscous fluid, Mathem. Models and Appl. 27 (2004), 1969-1995.

[12] C. W. Oseen, Neuere Methoden und Ergebnisse in der Hydrodynamik, Akad. Verlagsgesellschaft M.B.H., Leipzig, 1927.

[13] D. Smith, Estimates at infinity for stationary solutions of the N.S. equations in two dimensions, Arch. Rat. Mech. Anal. 20 (1965), 341-372.

[14] R. E. Showalter, Hilbert Space Methods for Partial Differential Equations, Pitman Publishing, San Francisco, 1979. 